\title{
Gene co-expression networks are associated with obesity-related traits in kidney transplant recipients
}

\author{
Rosario B. Jaime-Lara ${ }^{1 \dagger}$, Abhrarup Roy ${ }^{1 \dagger}$, Yupeng Wang ${ }^{2}$, Ansley Stanfill ${ }^{3}$, Ann K. Cashion ${ }^{1}$ and Paule V. Joseph ${ }^{1^{*}}$ (D)
}

\begin{abstract}
Background: Obesity is common among kidney transplant recipients; However biological mediators of obesity are not well understood in this population. Because subcutaneous adipose tissue can be easily obtained during kidney transplant surgery, it provides a unique avenue for studying the mechanisms of obesity for this group. Although differential gene expression patterns were previously profiled for kidney transplant patients, gene co-expression patterns can shed light on gene modules not yet explored on the coordinative behaviors of gene transcription in biological and disease processes from a systems perspective.
\end{abstract}

Methods: In this study, we collected 29 demographic and clinical variables and matching microarray expression data for 26 kidney transplant patients. We conducted Weighted Gene Correlation Network Analysis (WGCNA) for 5758 genes with the highest average expression levels and related gene co-expression to clinical traits.

Results: A total of 35 co-expression modules were detected, two of which showed associations with obesityrelated traits, mainly at baseline. Gene Ontology $(\mathrm{GO})$ enrichment was found for these two clinical traitassociated modules. One module consisting of 129 genes was enriched for a variety of processes, including cellular homeostasis and immune responses. The other module consisting of 36 genes was enriched for tissue development processes.

Conclusions: Our study generated gene co-expression modules associated with obesity-related traits in kidney transplant patients and provided new insights regarding the cellular biological processes underlying obesity in this population.

Keywords: Co-expression modules, Obesity, Association, Enrichment analysis, Gene ontology, Kidney transplant

\section{Background}

Obesity is a growing health concern worldwide and is associated with renal co-morbidities such as Chronic Kidney Disease (CKD), End Stage Renal Disease (ESRD), and other kidney complications [1-7]. In 2011, 23\% of kidney transplant recipients in the

\footnotetext{
* Correspondence: paule.joseph@nih.gov

${ }^{\dagger}$ Rosario B. Jaime-Lara and Abhrarup Roy are the co-first authorship ${ }^{1}$ Division of Intramural Research, National Institute of Nursing Research, National Institutes of Health, Bethesda, MD 20892, USA

Full list of author information is available at the end of the article
}

United States were obese, 9.4\% were morbidly obese, and $2.1 \%$ were morbidly obese [8]. In addition, posttransplantation weight gain in kidney transplant cohorts is common, thus exacerbating patients' preexisting obese phenotype and decreasing the likelihood of long-term renal allograft success $[9,10]$.

Obesity is influenced by the interaction of genetic and environmental factors [11-13], and as obesity prevalence increases and genetic methods evolve, there is a growing interest in studying the genetic

(c) The Author(s). 2020 Open Access This article is licensed under a Creative Commons Attribution 4.0 International License, which permits use, sharing, adaptation, distribution and reproduction in any medium or format, as long as you give appropriate credit to the original author(s) and the source, provide a link to the Creative Commons licence, and indicate if changes were made. The images or other third party material in this article are included in the article's Creative Commons licence, unless indicated otherwise in a credit line to the material. If material is not included in the article's Creative Commons licence and your intended use is not permitted by statutory regulation or exceeds the permitted use, you will need to obtain permission directly from the copyright holder. To view a copy of this licence, visit http://creativecommons.org/licenses/by/4.0/ The Creative Commons Public Domain Dedication waiver (http://creativecommons.org/publicdomain/zero/1.0/) applies to the data made available in this article, unless otherwise stated in a credit line to the data. 
and biological mechanisms driving weight gain. Several studies have investigated genetic mechanisms of obesity through various methodologies, including gene expression studies, Genome Wide Association Studies (GWAS), and obesity-related biomarkers [14, 15]. However, more comprehensive systems-based genetic analyses, which could provide a more robust understanding of gene interactions, pathways, and biological functions, are underexplored. Such inquiry could provide a more robust understanding of gene interaction networks, their pathways, and biological functions. Weighted gene correlation network analysis (WGCNA) is one such systems-based approach that offers the ability to create modules of gene networks (groups of co-expressed genes) that are highly associated with clinical variables of interest (e.g., Body Mass Index).

In addition to underutilizing systems-based approaches to study obesity, very few researchers have conducted genetic studies in human subcutaneous adipose tissue. Collecting human tissue, including adipose tissue, involves invasive procedures, making other noninvasive methods of sample collection (i.e., blood, stool, and saliva) more feasible and attractive to research participants. However, subcutaneous adipose tissue can be easily obtained during kidney transplant surgery. Furthermore, subcutaneous adipose tissue is a particularly promising candidate for gene studies of obesity, because it is metabolically active and plays an important role in endocrine pathways that modulate eating behavior and metabolism (e.g., appetite regulation, insulin signaling, and leptin signaling) [16].

A recent study by Joseph at al. carried out an analysis of gene co-expression patterns associated with body mass index using expression data in whole blood cells [17]. However, it is still unknown how gene co-expression patterns in adipose tissue are related to obesity. A longitudinal study by Cashion et al. analyzed gene expression data from a cohort of kidney transplant recipients to identify individual genes and molecular pathways that could be driving weight gain [18]. Differential gene expression analysis revealed that changes in gene expression were associated with insulin, inflammatory signaling pathways, and leptin. Their analysis also identified four obesityassociated genes (CPE, LEP, NPY1R, and NPY5R) that were positively correlated with weight gain and two genes (APOM and $C R P$ ) that were negatively correlated with weight gain. Cashion et al. also found demographic and environmental factors were associated with weight gain following kidney transplantation [19]. Although Cashion et al. compared expressed genes between those who did and did not gain weight, they did not analyze the interaction patterns among expressed genes. The current study builds on these previous findings by Cashion et al. and utilizes gene expression data to analyze gene co-expression networks to examine obesity-related traits in kidney transplant recipients [18].

\section{Methods \\ Materials and methods \\ Design and setting}

This study utilized gene expression data to examine gene interactions in human adipose tissue of kidney transplant recipients. Tissue samples were obtained from investigators at a regional MidSouth transplant center, who recruited 153 transplant recipients from 2006 to 2011 to study the genetic and environmental factors associated with weight gain following kidney transplantation. Written informed consent was obtained from all participants, and some patients also signed an optional repository consent. The parent study was approved by the Institutional Review Board of the University of Tennessee Health Science Center. Additional analyses on the repository samples were approved by the Office of Human Subjects Research and the Institutional Review Board of the National Institutes of Health.

All adults, regardless of race or sex, were eligible for participation. To control for the effect of pretransplantation immunosuppressant therapy on gene expression profiles, exclusion criteria included prior treatment with prednisone or other immunosuppressant therapies. Other exclusion criteria included underweight status (i.e., BMI $<18.5 \mathrm{~kg} / \mathrm{m}^{2}$ ) and pre-existing conditions that may impact weight, including gastrointestinal, pulmonary, neurologic, or gynecological diseases. Of the 153 transplant recipients, thirty signed a repository consent and also had RNA microarray data. Of these thirty participants, three were excluded due to excessive weight loss, which was determined to be a potential indicator of an abnormal recovery. An additional participants' sample was excluded as it did not meet quality control criteria. A total of 26 participant samples were included in the current study.

\section{Demographic and clinical variables}

Demographic data (age, race, and sex) were collected from electronic medical records (Table 1). Clinical laboratory values were collected at the time of transplantation and at 3,6 , and 12 months post transplantation. Clinical variables were analyzed including height, weight, BMI, body fat, creatinine levels, and glucose levels. Body weight and height were used to calculate BMI (weight in kilograms divided by height in meters squared). Weight categories were selected as recommended by the Centers for Disease Control, with greater than $30 \mathrm{~kg} / \mathrm{m}^{2}$ indicating obesity. Body fat was determined using dual-energy X-ray absorptiometry (DEXA) scans. Blood samples were collected to determine laboratory values (e.g., 
Table 1 Demographic characteristics of the study cohort ( $N=$

\begin{tabular}{ll}
$26)$ & \\
\hline Characteristic & Value \\
\hline Age at transplantation, mean (range) & 47.7 (19 67) \\
Sex, n (\%) & \\
$\quad$ Male & $11(42.3 \%)$ \\
Female & $15(57.7 \%)$ \\
Race, n (\%) & \\
African American & $15(57.7 \%)$ \\
Caucasian & $11(42.3 \%)$ \\
\hline
\end{tabular}

fasting glucose and creatinine). Reference values of normality for creatinine and fasting glucose levels were $0.5-1.2 \mathrm{mg} / \mathrm{dL}$ and $70-100 \mathrm{mg} / \mathrm{dL}$ respectively.

The baseline clinical variables included weight (WT_ BL), height, total subcutaneous body fat (SubTot_Fat_ $\mathrm{BL}$ ), percent of subcutaneous body fat (SubTot_Pfat BL), whole-body fat percent (WB_Tot_Pfat_BL), body mass index (BMI_BL), blood creatinine (CREATININE_ $\mathrm{BL}$ ), and blood glucose (GLUCOSE_BL). The following variables were also measured at 3, 6, and 12 months after kidney transplantation: blood creatinine (CREATININE_ 3M, CREATININE_6M, CREATININE_12M) and blood glucose (GLUCOSE_3M, GLUCOSE_6M, GLUCOSE_ $12 \mathrm{M})$. Other variables were measured at 12 months, including SubTot_Fat_12M, SubTot_Pfat_12M, and BMI_ $12 \mathrm{M}$.

Additionally, other pre-existing conditions (i.e. hyperlipidemia, hypertension, diabetes and depression) were also collected from the medical charts and controlled for in our analysis. Adipose tissue samples were collected for all eligible participants at the time of transplant surgery. Participants followed similar immunosuppressant therapy for 6 months following transplantation, 80\% received $20 \mathrm{mg}$ prednisone, $8 \%$ received $10 \mathrm{mg}$ of prednisone, and $4 \%$ received $50 \mathrm{mg}$ of prednisone. Six months after undergoing a kidney transplant, $92 \%$ received $5 \mathrm{mg}$ of prednisone and $8 \%$ did not take prednisone.

\section{Microarray data processing and annotation}

As a part of the parent study, RNA was extracted from adipose tissue samples and gene expression values were obtained by the authors and Cashion et al. [18]. For RNA extraction procedures please refer to Cashion et al. [18]. The expression values were generated by Affymetrix Human Gene 1.0 ST Array (GEO platform ID: GPL6244) and results were made available via GEO (https://www.ncbi.nlm.nih.gov/geo/, Dataset ID: GSE33070; annotation file: GPL6244.annot). These microarray data are publicly available, although due to privacy concerns only limited amount of demographic and clinical data for these subjects are publicly available. Such data were available to the authors on this paper through this current collaboration, though they are still limited to the repository dataset (specific available variables are described above).

The microarray data were annotated using custom programs to process the annotation file and to generate mapping between genes and transcript IDs. For each gene, we selected the transcript with the highest average expression level to represent the expression of that gene. Using this process, we obtained a total of 19,192 gene expression profiles.

\section{Constructing gene co-expression networks}

A total of 5758 (30\%) highly expressed genes were selected for co-expression network analysis. The WGCNA package was used to construct gene co-expression networks and examine their associations with clinical variables [20]. A soft-threshold power of 7 was used as it met scale-free topology criteria $\left(R^{2} \approx 0.8\right)$ while generating reasonable module sizes $($ mean $=37)$ (Fig. S1). Then, one-step network construction and module detection were performed with the following parameters: TOMType = "unsigned", minModuleSize $=10$, reassign Threshold $=0$, and mergeCutHeight $=0.25$. Next, for each detected gene module, its eigenvector (vectors associated with linear system equations) was computed. Module-trait associations were assessed based on the Pearson's correlation between the eigenvector of each module and each clinical variable. Original $p$-values of module-trait associations were adjusted for multiple testing using the Benjamini-Hochberg (i.e. False Discovery Rate or FDR) approach [21].

\section{Enrichment analysis}

Functional enrichment analysis was performed using the online version of GOStat (http://gostat.wehi.edu.au/ cgi-bin/goStat.pl) [22]. Parameters were chosen as follows- database: goa_human; minimal length of considered GO paths: 3 ; maximal $p$-value: 0.05; maximum number of GOs: 30; cluster GOs: 5; direction: overrepresented; correct for multiple testing: false discovery rate. The 5758 highly expressed genes were used as a genomic background to examine enrichment of coexpression gene modules.

\section{Identification of hub genes in co-expression gene modules}

Hub genes were identified using topology similarity as described previously by Joseph et al. [17]. Briefly, we used topology similarity to measure the comparability between gene expression profiles, and computed the topological matrix for the 5758 highly expressed genes. Subsequently, 95\% quantile of the topological matrix was used as the cutoff for determining whether two genes were connected. The connectivity of a gene was 
the number of genes connected with it in the same gene module. This hub gene identification method was custom built using $\mathrm{R}$ software. In our analysis, hub genes in co-expression gene modules were defined as genes with high connectivity (ranked at the top 10\%) in the candidate modules.

\section{Pathway analysis}

Ingenuity pathway analysis (IPA), https://www. qiagenbioinformatics.com/products/ingenuity-pathwayanalysis/ was used for functional analysis, integration, and interpretation of the biological role of the magenta and darkgreen modules. One hundred twenty-nine genes within the magenta module and 36 genes within the darkgreen module were input separately into IPA and a "Core Analysis" was used to construct canonical pathways and networks. Canonical pathways were constructed to examine the role of genes within each module (i.e. their role in cell signaling and metabolic pathways). Networks were constructed to identify and visualize gene-gene interactions, each connection representing known relationships between genes. Identified networks were scored based on the degree of relevance (i.e. - $\log$ (Fisher's Exact test)) of their genes with a list of biological functions stored in the Ingenuity Knowledge Base. The Human Gene 1.0 ST Array was used as the reference background in order to reduce bias towards pathways identified in the default of option which uses the entire "Ingenuity Knowledge Base (Genes Only)" as a reference set. Additionally we ran the 5758 highly expressed genes separately and compared the results. We report the findings, canonical pathways and networks, for the module results.

\section{Results}

\section{Study data}

The demographic characteristics of our 26 kidney transplant recipients are summarized in Table 1. The clinical characteristics for these same recipients are shown in Table 2. A total of 26 different clinical variables were measured.

The adipose tissue samples were used to generate raw RNA expression data as a part of a previous study [18]. These samples were taken at the time of transplantation surgery, and thus expression data were available for each subject at baseline. We further processed the existing RNA expression data to generate expression profiles for a total of 19,192 genes (see Methods).

In the original study dataset, associations between gene expression and weight change were controlled for sex and race. Weight/height traits were frequently related to demographic characteristics $[18,23]$. To more accurately control for age, sex, and race as correlated to weight and height traits, we computed the association levels between each of the demographic and clinical variables (Table 3). Using this method, when an association between demographic and clinical variables was noted, it was controlled for demographic characteristics. We found that weight change variables were not associated with any demographic variable, and age was not associated with any weight and height trait in this study. Height, weight, and BMI variables showed significant associations with sex at all time points (baseline, and 3, 6, and 12 months) and with race at baseline. Therefore, when we calculated the association between eigenvectors and clinical variables, we adjusted height/weight traits for sex and race at baseline and for sex at other time points.

We further assessed whether gene expression data could be affected by confounding factors at baseline. To this end, we computed the first three principal components of gene expression data and related them to demographic characteristics (gender, age group and race), clinical parameters (glucose and creatinine), and preexisting diseases (hyperlipidemia, hypertension, diabetes and depression). Ages were divided into binary groups by age (above or below age 50). Age was divided into two even groups (above or below age 50) in order to better compare age with sex and race (both categorical variables). Additionally, age was tested as a continuous variable and as a continuous variable age was not significantly correlated with the first three principal components of microarray data. For each discrete variable, an ANOVA model was fit between the first three principal components and the variable. For each quantitative variable, a linear regression model was fit between the first three principal components and the variable. The $p$ values for all variables were retrieved and adjusted for multiple testing according to the Benjami-Hochberg procedure [21]. The outcome (Supplementary Table S1) shows that none of these variables is significantly associated with the first three principal components of the gene expression data. This analysis suggests that potential confounding factors are not substantially affecting gene expression analysis.

\section{Gene co-expression networks and their relationship to obesity traits and fat-related traits in kidney transplant patients}

Gene co-expression networks can reveal coordinative behaviors of gene transcription for diseases or medical interventions. We conducted Weighted Gene Correaltion Network Analysis (WGCNA) to obtain a better understanding of gene expression mechanisms underlying obesity-related traits. 5758 (30\%) of the most highly expressed genes were examined to reduce the noise (i.e., genes not actually expressed). Fitting of scale-free topology identified 35 gene modules with sizes ranging from 10 to 1451 genes and a median size of 49 (Fig. 1). We 
Table 2 Clinical characteristics of the study cohort $(\mathrm{N}=26)$

\begin{tabular}{|c|c|c|}
\hline Clinical parameter & Mean \pm Standard deviation & Range (min; max) \\
\hline \multicolumn{3}{|l|}{ Weight at Baseline } \\
\hline WT_BL (lbs) & $174.80 \pm 37.54$ & $129.19 ; 257.90$ \\
\hline \multicolumn{3}{|l|}{ Weight at 3 Months } \\
\hline WT_3M (lbs) & $170.22 \pm 38.79$ & $123.00 ; 242.00$ \\
\hline \multicolumn{3}{|l|}{ Weight at 6 Months } \\
\hline WT_6M (lbs) & $179.96 \pm 39.91$ & $133.00 ; 259.80$ \\
\hline \multicolumn{3}{|l|}{ Weight at 12 Months } \\
\hline WT_12M (lbs) & $182.69 \pm 47.33$ & $128.00 ; 294.62$ \\
\hline \multicolumn{3}{|l|}{ BMI at Baseline } \\
\hline BMI_BL $\left(\mathrm{kg} / \mathrm{m}^{2}\right)$ & $27.41 \pm 3.36$ & $22.30 ; 33.62$ \\
\hline \multicolumn{3}{|l|}{ BMl at 3 Months } \\
\hline BMI_3M $\left(\mathrm{kg} / \mathrm{m}^{2}\right)$ & $26.68 \pm 3.82$ & $20.52 ; 34.85$ \\
\hline \multicolumn{3}{|l|}{ BMl at 6 Months } \\
\hline BMI_6M $\left(\mathrm{kg} / \mathrm{m}^{2}\right)$ & $28.21 \pm 3.73$ & $22.30 ; 34.70$ \\
\hline \multicolumn{3}{|l|}{$\mathrm{BMI}$ at 12 Months } \\
\hline BMI_12M (kg/m²) & $28.58 \pm 4.97$ & $22.64 ; 39.95$ \\
\hline \multicolumn{3}{|l|}{ Weight Change at 3 Months } \\
\hline WT_CHG_3M (\%) & $-2.71 \pm 6.10$ & $-13.92 ; 8.13$ \\
\hline \multicolumn{3}{|l|}{ Weight Change at 6 Months } \\
\hline WT_CHG_6M (\%) & $3.01 \pm 6.69$ & $-12.02 ; 14.24$ \\
\hline \multicolumn{3}{|l|}{ Weight Change at 12 Months } \\
\hline WT_CHG_12M (\%) & $4.11 \pm 10.83$ & $-17.00 ; 25.84$ \\
\hline \multicolumn{3}{|l|}{ Subtotal Body Fat at Baseline } \\
\hline SubTot_Fat_BL (g- below the head) & $22,006.51 \pm 7037.55$ & $7505.83 ; 35,429.71$ \\
\hline \multicolumn{3}{|l|}{ Percent Body Fat at Baseline } \\
\hline SubTot_Pfat_BL (\%) & $29.79 \pm 7.39$ & $14.05 ; 38.47$ \\
\hline \multicolumn{3}{|l|}{ Whole Body Fat at Baseline } \\
\hline WB_Tot_Pfat_BL (g) & $29.25 \pm 6.95$ & $14.50 ; 37.60$ \\
\hline \multicolumn{3}{|l|}{ Subtotal Body Fat at 12 Months } \\
\hline SubTot_Fat_12M ( $\mathrm{g}$ - below the head) & $26,416.99 \pm 9604.71$ & $14,528.39 ; 49,349.50$ \\
\hline \multicolumn{3}{|l|}{ Percent Body Fat at 12 Months } \\
\hline SubTot_Pfat_12M (\%) & $34.07 \pm 6.85$ & $19.20 ; 50.19$ \\
\hline \multicolumn{3}{|l|}{ Whole Body Fat at 12 Months } \\
\hline WB_Tot_Pfat_12M (\%) & $33.28 \pm 6.52$ & $19.25 ; 48.84$ \\
\hline \multicolumn{3}{|l|}{ Creatininie at Baseline } \\
\hline CREATININE_BL (mg/dL) & $6.24 \pm 3.60$ & $1.20 ; 14.10$ \\
\hline \multicolumn{3}{|l|}{ Glucose at Baseline } \\
\hline GLUCOSE_BL (mg/dL) & $116.50 \pm 44.23$ & $70.00 ; 234.00$ \\
\hline \multicolumn{3}{|l|}{ Creatinine at 3 Months } \\
\hline CREATININE_3M (mg/dL) & $1.58 \pm 0.42$ & $1.00 ; 2.50$ \\
\hline \multicolumn{3}{|l|}{ Glucose at 3 months } \\
\hline GLUCOSE_3M (mg/dL) & $125.68 \pm 51.46$ & $70.00 ; 266.00$ \\
\hline \multicolumn{3}{|l|}{ Creatininte at 6 Months } \\
\hline CREATININE_6M (mg/dL) & $1.42 \pm 0.28$ & $1.00 ; 1.90$ \\
\hline
\end{tabular}


Table 2 Clinical characteristics of the study cohort $(N=26)$ (Continued)

\begin{tabular}{llc}
\hline Clinical parameter & Mean \pm Standard deviation & Range (min; max) \\
\hline Glucose at 6 Months & & $121.48 \pm 37.72$ \\
$\quad$ GLUCOSE_6M (mg/dL) & & $79.00 ; 224.00$ \\
Creatinine at 12 Months & $1.60 \pm 0.45$ & $0.85 ; 2.68$ \\
$\quad$ CREATININE_12M (mg/dL) & & $78.00 ; 285.00$ \\
Glucose at 12 Months & $135.29 \pm 59.50$ & $60.00 ; 75.00$ \\
$\quad$ GLUCOSE_12M (mg/dL) & $66.61 \pm 4.07$ & \\
Height (in) & &
\end{tabular}

then examined associations between these 35 gene modules and 26 clinical variables (see Methods, Fig. 2). In order to ensure detection of reliable associations, the $p$ values obtained from the WGCNA software were further adjusted for multiple testing according to the BenjamiHochberg approach. At an adjusted $p$-value cutoff of 0.05 , two modules showed association with at least one clinical trait: the "magenta" module was correlated with SubTot_Fat_BL; the "darkgreen" module was correlated with SubTot_Pfat_BL and WB_Tot_Pfat_BL.

For each module, the eigengene represents its major gene expression pattern among its component gene members. The gene module membership measures how close a gene's expression profile is to its eigengene, and it is expected that genes with higher module memberships are more functionally important in the module and are more likely to be correlated with clinical traits. In turn, if genes in a module show high correlations between module memberships and gene significance (for association with traits), that suggests that the association between the gene module and the clinical trait is highly reliable. To this end, we made plot gene module memberships and gene significance for the modules associated with clinical traits (Fig. 3). The magenta module showed a highly significant module-membership to gene-significance correlation with the SubTot_Fat_BL trait. The darkgreen module held moderate module-membership to genesignificance correlations with SubTot_Pfat_BL and WB_Pfat_BL. This analysis confirmed that both the magenta and darkgreen modules are functionally important for obesity-related traits.

\section{Functional landscapes of obesity-related gene modules}

We then utilized GO enrichment analysis to examine the association between functional properties and clinical traits within the magenta and darkgreen modules. Both modules showed GO enrichment at an FDR-corrected $p$-value of 0.05 . The magenta module contains 129 genes. The GO term enrichment for the co-expression modules is shown in supplementary Table S2. GO enrichment of the magenta module identified multiple biological components and processes associated with SubTot_Fat_BL, including

Table 3 Assessment of associations between clinical and demographic characteristics

\begin{tabular}{llll}
\hline Clinical characteristics & Association ( -value) & \\
\cline { 2 - 4 } & $\begin{array}{l}\text { Age at Transplantation (by linear } \\
\text { regression) }\end{array}$ & $\begin{array}{l}\text { Gender (by Analysis of } \\
\text { Variance) }\end{array}$ & $\begin{array}{l}\text { Race (by Analysis of } \\
\text { Variance) }\end{array}$ \\
\hline Height & 0.542 & $1.22 \times 10^{-5}$ & 0.282 \\
Weight, baseline & 0.737 & $4.59 \times 10^{-6}$ & 0.049 \\
Weight at 3 months & 0.672 & $3.25 \times 10^{-5}$ & 0.066 \\
Weight at 6 months & 0.939 & $6.66 \times 10^{-6}$ & 0.088 \\
Weight at 12 months & 0.991 & $7.38 \times 10^{-5}$ & 0.216 \\
BMl at baseline & 0.235 & $2.33 \times 10^{-3}$ & 0.037 \\
BMl at 3 months & 0.242 & 0.013 & 0.074 \\
BMl at 6 months & 0.643 & $3.36 \times 10^{-3}$ & 0.121 \\
BMl at 12 months & 0.697 & 0.011 & 0.429 \\
Weight change percentage at 3 months & 0.739 & 0.928 & 0.849 \\
Weight change percentage at 6 months & 0.276 & 0.778 & 0.542 \\
Weight change percentage at 12 & 0.476 & 0.442 & 0.292 \\
months & & & \\
\hline
\end{tabular}




\section{Cluster Dendrogram}

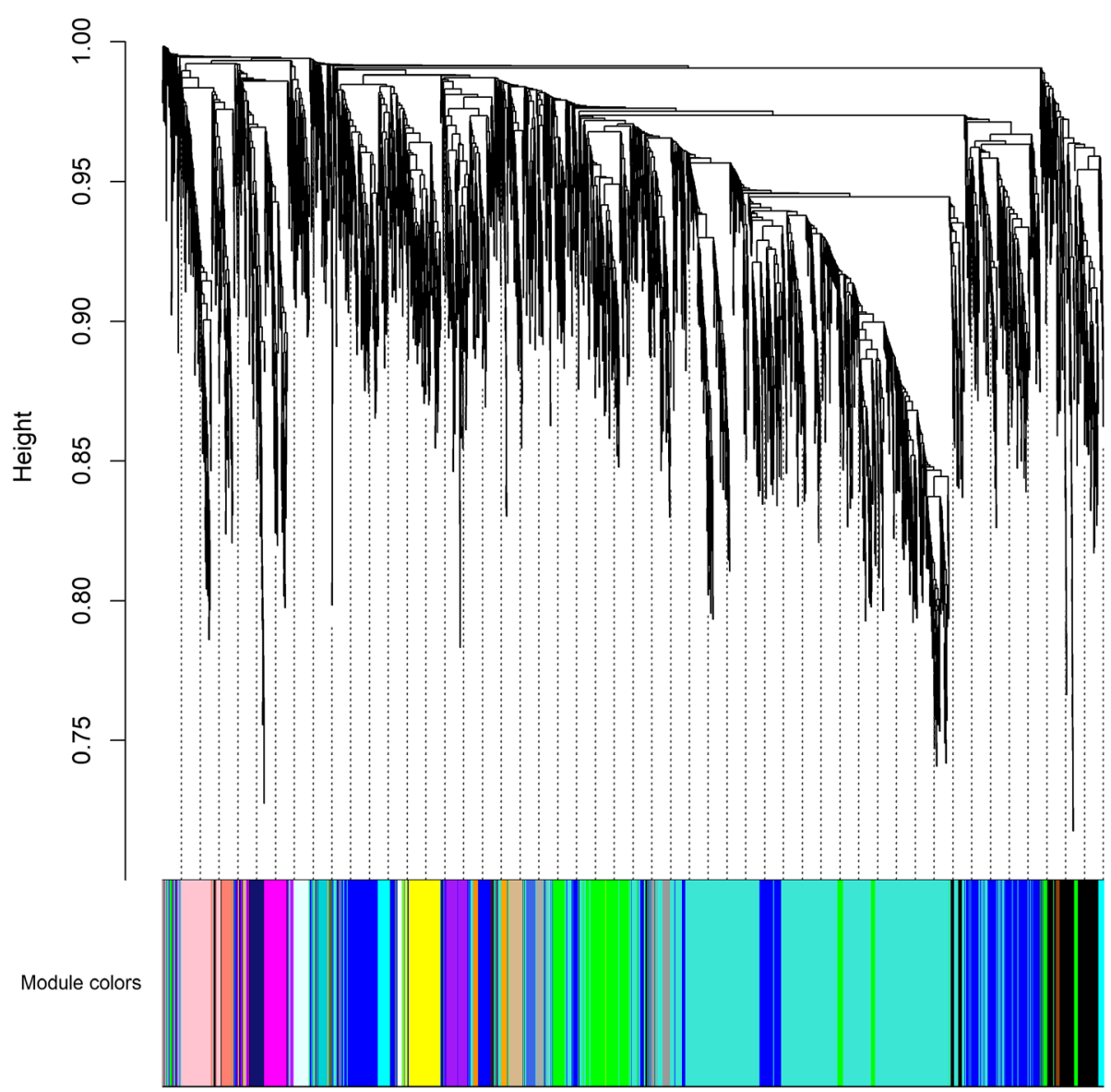

Fig. 1 Dendrogram showing the module-gene relationships generated by the WGCNA software

immune system processes and lipid metabolism. Enrichment of the darkgreen module using GOStat found an association of SubTot_Pfat_BL and WB Tot_Pfat_BL with heart and vasculature development.

To further explore the functionality of the magenta and darkgreen module, we classified the top $10 \%$ of genes with the highest connectivity as hub genes. The top 2 hub genes within the magenta module were ITGAM (92 connections), CD68 (89 connections). The top 2 hub genes within the darkgreen module were CRLS1 (18 connections) and ACSS3 (12 connections).

IPA-identified canonical pathways and gene networks within the magenta and darkgreen module. Canonical pathways within the magenta module included: phagosome maturation, autophagy, lipid antigent presentation, and inflammatory signaling (e.g. Il-6 and Il-8) (Fig. 4). Eight networks where identified in the magenta module; Top networks were associated with immune cell trafficking (score 12), cardiovascular disease (score 18), lipid metabolism (score 22), and inflammatory response (score 11). Canonical pathways within the darkgreen module included: hypoxia signaling in the cardiovascular system, cardiac hypertrophy signaling, and the Methylmalonyl Pathway. Top networks in the darkgreen module were associated with infectious diseases (score 30), gastrointestinal disease (score 27), and cardiovascular system development and function (score 22).

\section{Discussion}

This study sought to identify sets of genes that could provide insight into the genetic underpinnings of obesity-related traits in kidney transplant recipients. Using gene expression data from adipose tissue of kidney transplant recipients, we created gene co-expression networks and correlated them to clinical variables. Previous work has identified individual genes associated with weight gain in kidney transplant populations [18, 23], but to our knowledge no studies have incorporated WGCNA in similar cohorts. Using WGCNA, we found two modules, the magenta module and the darkgreen module, to be significantly correlated with obesityrelated traits at baseline. 


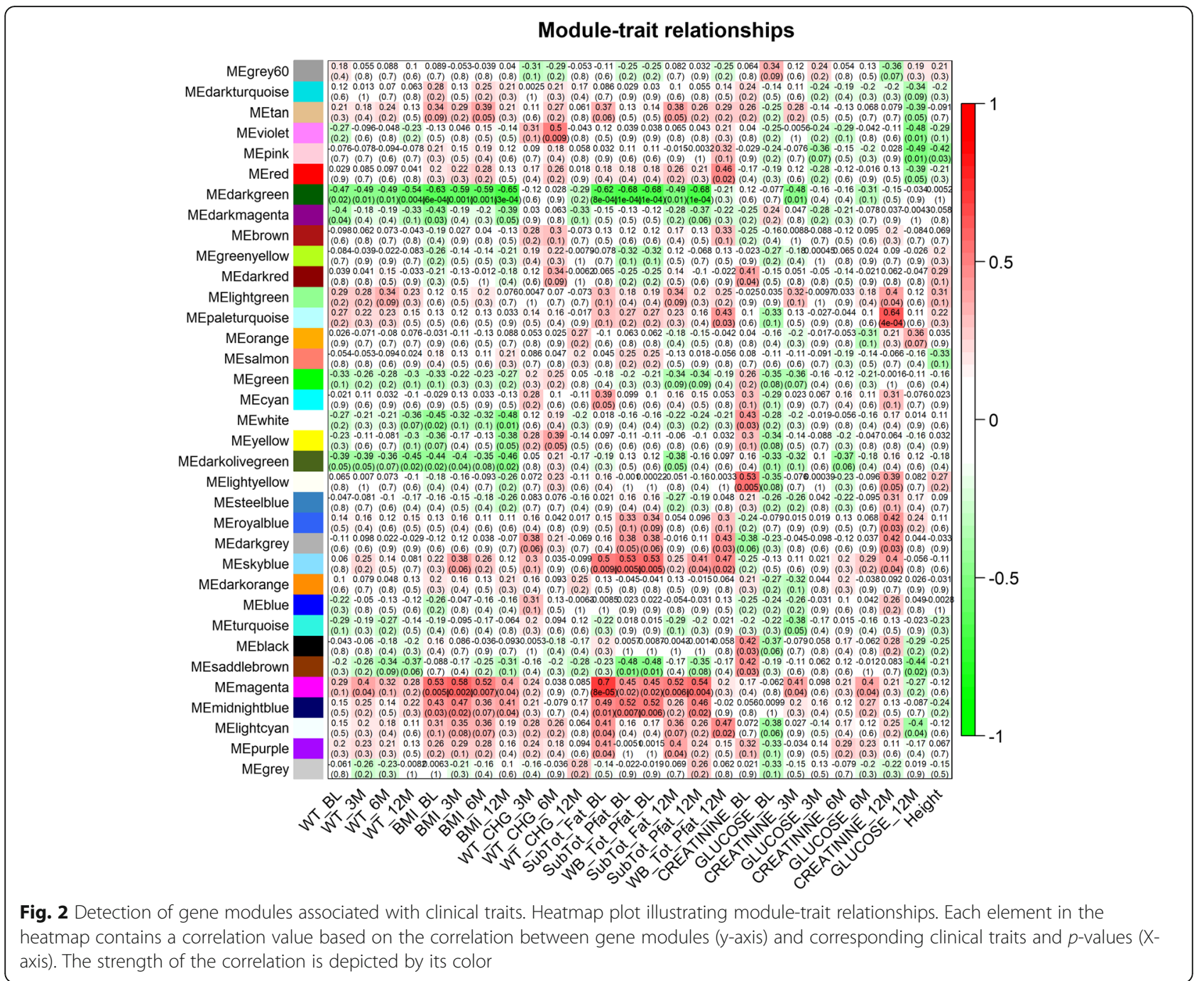

GO enrichment analysis and identification of hub genes revealed plausible biological functions of genes within the magenta and darkgreen modules. GO enrichment analysis identified that among the 129 genes comprising the magenta model, multiple genes are involved in immune function, inflammation, lipid metabolism, and cardiovascular disease. This is supported by the role of the hub genes within the magenta module. As mentioned previously, the top two hub genes in the magenta module are ITGAM and CD68. ITGAM plays a role in the adhesion of monocytes, macrophages, and granulocytes and the uptake of pathogens [24, 25]. CD68 codes for a glycoprotein expressed in monocytes and macrophages and is related to the innate immune system and low-density lipoprotein (LDL) oxidation in Atherogenesis [26, 27]. Thus, GO enrichment and hub genes are consistent, both suggesting the magenta module may hold immune-related, metabolic, and cardiovascular roles in kidney transplant patients.
The biological processes identified by GO enrichment and the hub genes' function are also consistent with our network analysis using IPA. Network analysis of the magenta module revealed that 15 genes within this module, including ITGAM and CD68, have immune-related functions. Additionally, within the magenta module, IPA identified 2 networks associated with lipid metabolism (22 genes) and metabolic disease (11 genes). Other genes within the magenta module play a role in metabolic functions and obesity including genes that code for lipase (LIPA), phospholipid transfer protein (PTLP), [28] and lipid metabolism (e.g., LPIN1) [29]. This suggests that there is a relationship between metabolism, immune response, inflammatory-related genes, and body-fat in kidney transplant patients.

Like the magenta module, the darkgreen module contains genes associated with lipid metabolism and cardiovascular processes. The darkgreen module was enriched for cardiovascular processes including heart and vasculature 


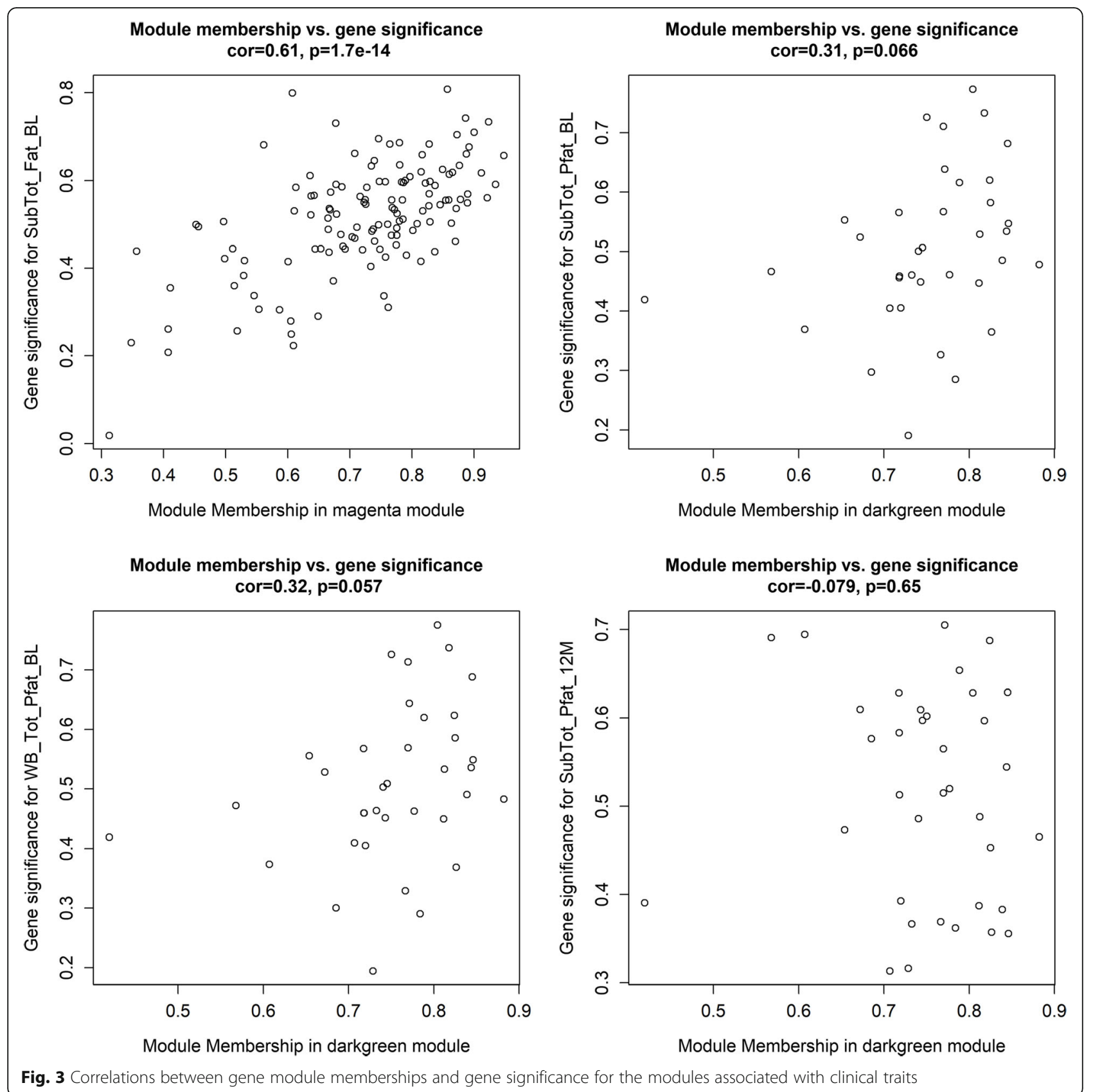

development. Additionally, the darkgreen module contains genes associated with cellular energy expenditure (e.g. mitochondrion and sodium: potassium-exchanging ATPase activity). The top 2 hubgenes, CRLS1 and ACSS3 are involved in lipid metabolism, including the catalysis of key phospholipids and acetyl-CoA from short-chain fatty acids [30,31]. Network analysis of the darkgreen module was also consistent with the GO enrichment analysis and the function of the hub genes, containing three networks associated with the immune response (e.g. infectious diseases), and cardiovascular processes (e.g. cardiovascular system development and function). Thus, body-fat associated genes within the darkgreen module are associated with cardiovascular health, lipid metabolism, and cellular energy expenditure. Both modules from our analysis are tied to immunological responses such as inflammation, suggesting the immune system may play a role in regulating obesity-related traits (e.g. total subcutaneous body fat) in kidney transplant patients.

The findings of the current study are supported by previous studies which have found immunological, inflammatory, and metabolic genetic drivers are influential in the determination of obesity [32-34]. Only one previous study by Muniandy et al. used adipose tissue 

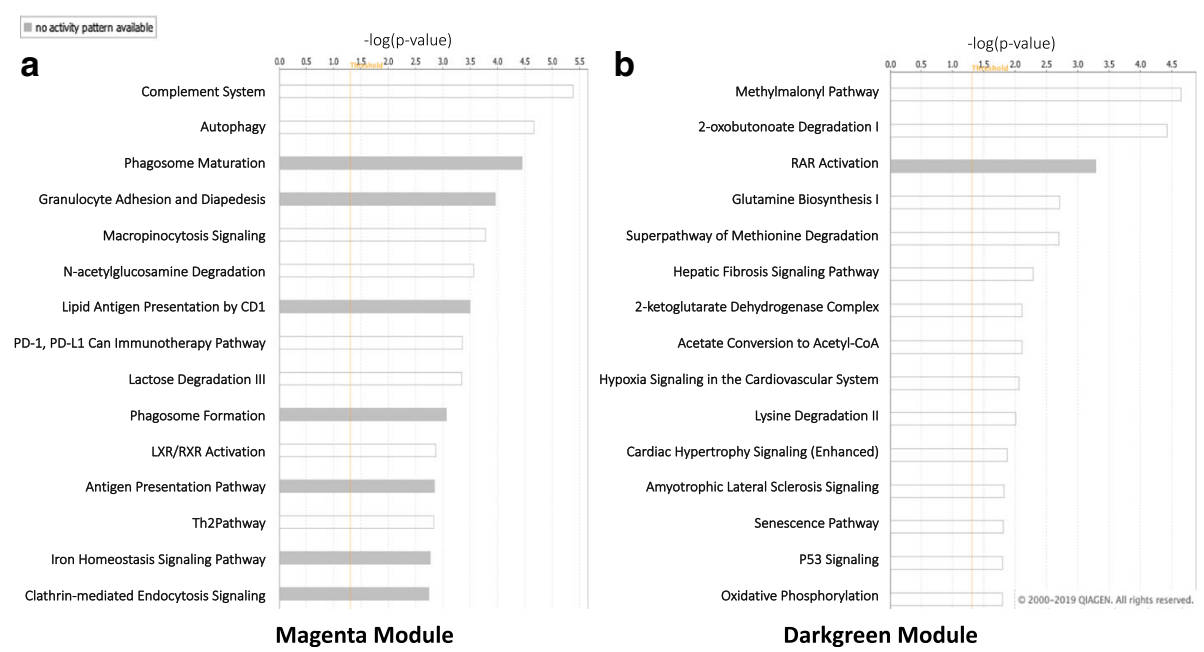

Fig. 4 Top canonical pathways for magenta and darkgreen modules generated by IPA. a Magenta canonical pathway. b Darkgreen canonical pathway

(subcutaneous) to study clinical changes associated with obesity; The study found that the heavier twin in BMIdiscordant monozygotic twin pairs displayed an upregulation of inflammation and other immunological pathways/genes (e.g. IFI30 and CCL18) [32]. Other studies have used blood samples to examine differential gene expression and weighted gene co-expression network analysis from a cohort of monozygotic twins [27]. A study by Wang et al. found 32 differentially expressed genes (DEGs) that were upregulated in the higher BMI twins including DEGs associated with obesity (e.g. NAMPT, TLR9, PTGS2, HBD, and PCSK1N), immunological (e.g., TLR9), and metabolic functions (e.g., PTGS2). Wang et al. also used WGCN to identify two modules strongly correlated to BMI including genes responsible for regulation of phospholipase activity, high density lipoprotein particle clearance, and voltage-gated potassium channel complex. Animal studies have also found that an association between obesity-related genes and inflammation. Studies have found inflammatory signaling affects adipocyte insulin receptors and causes insulin resistance, which further contributed to fatty acid accumulation and obesity $[35,36]$.

Gene modules were constructed based on gene expression at baseline. As these modules were constructed at baseline, we did not observe significant associations between gene modules and clinical traits beyond the baseline (i.e. following kidney transplant). As presented by Cashion et al. additional variables (aside from gene associations) contribute to clinical traits following transplantation [19]. For example younger age, higher carbohydrate consumption, higher trunk fat, and higher perception of mental health quality of life are predictors of weight gain following kidney transplantation [19].
Importantly, our analysis is limited to the clinical and demographic variables collected in the parent study. Additionally, only a portion of the participants in the parent study signed the repository consent and had available microarray data. Future studies should examine the co-expression patterns in larger more diverse populations of kidney transplant patients. Studies must also examine whether the obesity-related gene co-expression patterns observed in this study apply to weight gain in the general population.

\section{Conclusion}

The current study examined gene co-expression patterns associated with obesity-related traits in kidney transplant recipients. Utilizing WGCNA, we generated gene coexpression networks associated with obesity-related traits to highlight possible gene modules responsible for obesity, and assessed these modules' pathways, molecular functions, and gene-gene interactions with obesity-related traits. A total of 35 co-expression modules were detected, two modules were associated with clinical traits. These modules are involved in metabolic and immune processes (including genes involved in lipid metabolism and immune-related functions) and are associated with multiple obesity-related traits, total subcutaneous body fat and whole-body fat percent. This study offers a deeper understanding of the gene network properties underlying obesity-related traits and provides new insights regarding the biological processes underlying obesity in kidney transplant patients.

\section{Supplementary information}

Supplementary information accompanies this paper at https://doi.org/10 1186/s12920-020-0702-5.

Additional file 1: Fig. S1. Scale-Free Topology Model Fit. 
Additional file 2: Table S1. FDR-adjusted $p$-values of ANOVA/linear regression modules between the first three components of gene expression data and demographic/disease characteristics at baseline. Table S2. Enriched GO terms in the clinical trait-associated modules.

\section{Abbreviations}

BMI_BL: Body mass index at base line; CKD: Chronic Kidney Disease; CREATININE_12M: creatinine 12 months following transplantation; CREATININE_3M: creatinine 3 months following transplantation; CREATININE_6M: creatinine 6 months following transplantation; CREATININE BL: creatinine at baseline; DEXA: Dual-energy X-ray absorptiometry; ESRD: End Stage Renal Disease; GLUCOSE_12M: Glucose 3 months following transplantation; GLUCOSE_3M: Glucose 3 months following transplantation; GLUCOSE_6M: Glucose 3 months following transplantation; GLUCOSE_BL: Glucose at baseline; GO: Gene Ontology; GWAS: Genome Wide Association Studies; SubTot_Fat_BL: Subcutaneous body fat at baseline; SubTot_Pfat_BL: Percent of subcutaneous body fat at baseline; WB_Tot_Pfat_BL: Whole-body fat percent at baseline; WGCNA: Weighted Gene Correlation Network Analysis; WT_BL: Weight at baseline

\section{Acknowledgements}

The authors would like to thank Dr. Joan Austin for her comments and editorial assistance.

\section{Authors' contributions}

PVJ: contributed to study concept and design, acquisition, analysis and interpretation of data, manuscript development and critical revision of manuscript for intellectual content. YW: contributed to microarray data analysis, enrichment analysis, interpretation and manuscript development. RBJ \& AR: contributed to pathway and enrichment analysis, interpretation and manuscript development. AKC \& AS: study concept and design, study supervision, and critical revision of manuscript for intellectual content. All of the authors have approved the final submitted draft of the manuscript.

\section{Funding}

PVJ is supported by the National Institute of Nursing Research (1ZIANR000035-01), the Office of Workforce Diversity and the National Institutes of Health Distinguished Scholars Award, and by the Rockefeller University Heilbrunn Nurse Scholar Award. AKC is supported by the National Institute of Nursing Research (1ZIANR000030). The parent study by AKC NIH/ NINR5R01NR009270 is titled "Genetics, Environment and Weight Gain Posttransplant". RJL \& AR received Intramural Research Training Award, National Institute of Nursing Research, National Institutes of Health, Department of Health and Human Services. The content is solely the responsibility of the authors and does not necessarily represent the official views of the $\mathrm{NIH}$.

\section{Availability of data and materials}

Microarry data is available at the National Center for Biotechnology Information's Gene Exression Omnibus (GEO) Database at accession number GSE33070 https://www.ncbi.nlm.nih.gov/geo/query/acc.cgi?acc=GSE33070. Additional data is available upon request from senior author paule. joseph@nih.gov.

\section{Ethics approval and consent to participate}

The parent study was approved by the Institutional Review Board of the University of Tennessee Health Science Center. The additional analyses on the repository samples was approved by the Office of Human Subjects Research and the Institutional Review Board of the National Institutes of Health. Written informed consent was obtained from all participants, and some patients also signed an optional repository consent.

\section{Consent for publication}

Not applicable.

\section{Competing interests}

Potential competing interests: The authors have no potential conflicts of interest to disclose.

\section{Author details}

'Division of Intramural Research, National Institute of Nursing Research, National Institutes of Health, Bethesda, MD 20892, USA. 'BDX Research \& Consulting LLC, Herndon, VA 20171, USA. ${ }^{3}$ The University of Tennessee Health Science Center, College of Nursing, Memphis, Memphis, TN 38163, USA.

\section{Received: 22 August 2019 Accepted: 27 February 2020}

Published online: 10 March 2020

\section{References}

1. Tsujimoto T, Sairenchi T, Iso H, Irie F, Yamagishi K, Watanabe H, et al. The dose-response relationship between body mass index and the risk of incident stage $>/=3$ chronic kidney disease in a general japanese population: the Ibaraki prefectural health study (IPHS). J Epidemiol. 2014; 24(6):444-51.

2. Elsayed EF, Sarnak MJ, Tighiouart H, Griffith JL, Kurth T, Salem DN, et al. Waist-to-hip ratio, body mass index, and subsequent kidney disease and death. Am J Kidney Dis. 2008:52(1):29-38.

3. Munkhaugen J, Lydersen S, Wideroe TE, Hallan S. Prehypertension, obesity, and risk of kidney disease: 20-year follow-up of the HUNT I study in Norway. Am J Kidney Dis. 2009:54(4):638-46.

4. Iseki K, Ikemiya Y, Kinjo K, Inoue T, Iseki C, Takishita S. Body mass index and the risk of development of end-stage renal disease in a screened cohort. Kidney Int. 2004;65(5):1870-6.

5. Vivante A, Golan E, Tzur D, Leiba A, Tirosh A, Skorecki K, et al. Body mass index in 1.2 million adolescents and risk for end-stage renal disease. Arch Intern Med. 2012;172(21):1644-50.

6. Hsu CY, McCulloch CE, Iribarren C, Darbinian J, Go AS. Body mass index and risk for end-stage renal disease. Ann Intern Med. 2006;144(1):21-8.

7. Foster MC, Hwang SJ, Massaro JM, Hoffmann U, DeBoer IH, Robins SJ, et al. Association of subcutaneous and visceral adiposity with albuminuria: the Framingham Heart Study. Obesity (Silver Spring, Md). 2011;19(6):1284-9.

8. Lentine KL, Delos Santos R, Axelrod D, Schnitzler MA, Brennan DC, TuttleNewhall JE. Obesity and kidney transplant candidates: how big is too big for transplantation? Am J Nephrol. 2012;36(6):575-86.

9. de Vries AP, Bakker SJ, van Son WJ, van der Heide JJ, Ploeg RJ, The HT, et al. Metabolic syndrome is associated with impaired long-term renal allograft function; not all component criteria contribute equally. Am J Transplant. 2004;4(10):1675-83.

10. Hoogeveen EK, Aalten J, Rothman K, Roodnat JI, Mallat MJ, Borm G, et al. Effect of obesity on the outcome of kidney transplantation: a 20-year follow-up. Transplantation. 2011;91(8):869-74.

11. Martinez JA, Corbalan MS, Sanchez-Villegas A, Forga L, Marti A, MartinezGonzalez MA. Obesity risk is associated with carbohydrate intake in women carrying the GIn27Glu beta2-adrenoceptor polymorphism. J Nutr. 2003; 133(8):2549-54.

12. Santos JL, Boutin P, Verdich C, Holst C, Larsen LH, Toubro S, et al. Genotypeby-nutrient interactions assessed in European obese women. A case-only study. Eur J Nutr. 2006;45(8):454-62.

13. Sacks FM, Bray GA, Carey VJ, Smith SR, Ryan DH, Anton SD, et al. Comparison of weight-loss diets with different compositions of fat, protein, and carbohydrates. N Engl J Med. 2009;360(9):859-73.

14. Frayling $T M$, Timpson $N J$, Weedon $M N$, Zeggini E, Freathy RM, Lindgren $C M$, et al. A common variant in the FTO gene is associated with body mass index and predisposes to childhood and adult obesity. Science. 2007; 316(5826):889-94.

15. Sonne SB, Yadav R, Yin G, Dalgaard MD, Myrmel LS, Gupta R, et al. Obesity is associated with depot-specific alterations in adipocyte DNA methylation and gene expression. Adipocyte. 2017;6(2):124-33.

16. del Pozo CH, Calvo RM, Vesperinas-Garcia G, Gomez-Ambrosi J, Fruhbeck G, Rubio MA, et al. Expression profile in omental and subcutaneous adipose tissue from lean and obese subjects. Repression of lipolytic and lipogenic genes. Obes Surg. 2011;21(5):633-43.

17. Joseph PV, Jaime-Lara RB, Wang Y, Xiang L, Henderson WA. Comprehensive and systematic analysis of gene expression patterns associated with body mass index. Sci Rep. 2019;9(1):7447.

18. Cashion A, Stanfill A, Thomas F, Xu L, Sutter T, Eason J, et al. Expression levels of obesity-related genes are associated with weight change in kidney transplant recipients. PLoS One. 2013;8(3):e59962. 
19. Cashion AK, Hathaway DK, Stanfill A, Thomas F, Ziebarth JD, Cui Y, et al. Pretransplant predictors of one yr weight gain after kidney transplantation. Clin Transpl. 2014;28(11):1271-8.

20. Langfelder P, Horvath S. WGCNA: an R package for weighted correlation network analysis. BMC Bioinformatics. 2008;9:559.

21. Benjamini Y, Hochberg Y. Controlling the false discovery rate: a practical and powerful approach to multiple testing. J Royal Stat Soc. 1995;57(1):289300.

22. Falcon S, Gentleman R. Using GOstats to test gene lists for $\mathrm{GO}$ term association. Bioinformatics. 2007;23(2):257-8.

23. Stanfill A, Hathaway D, Cashion A, Homayouni R, Cowan P, Thompson C, et al. A pilot study of demographic and dopaminergic genetic contributions to weight change in kidney transplant recipients. PLoS One. 2015;10(9): e0138885

24. DiScipio RG, Daffern PJ, Schraufstatter IU, Sriramarao P. Human polymorphonuclear leukocytes adhere to complement factor $\mathrm{H}$ through an interaction that involves alphaMbeta2 (CD11b/CD18). J Immunol. 1998; 160(8):4057-66.

25. Losse J, Zipfel PF, Jozsi M. Factor $\mathrm{H}$ and factor H-related protein 1 bind to human neutrophils via complement receptor 3 , mediate attachment to Candida albicans, and enhance neutrophil antimicrobial activity. J Immunol. 2010;184(2):912-21.

26. Mazur G, Halon A, Wrobel T, Kuliczkowski K. Macrophage/histiocytic antigen CD68 expression in neoplastic and reactive lymph nodes. Rocz Akad Med Bialymst. 2004;49(Suppl 1):73-5.

27. van der Kooij MA, von der Mark EM, Kruijt JK, van Velzen A, van Berkel TJ, Morand $\mathrm{OH}$. Human monocyte-derived macrophages express an approximately 120-kD ox-LDL binding protein with strong identity to CD68. Arterioscler Thromb Vasc Biol. 1997;17(11):3107-16.

28. Koch G, Lalley PA, McAvoy M, Shows TB. Assignment of LIPA, associated with human acid lipase deficiency, to human chromosome 10 and comparative assignment to mouse chromosome 19. Somatic cell Genet. 1981;7(3):345-58.

29. Romani P, Brian I, Santinon G, Pocaterra A, Audano M, Pedretti S, et al. Extracellular matrix mechanical cues regulate lipid metabolism through Lipin-1 and SREBP. Nat Cell Biol. 2019;21(3):338-47.

30. Houtkooper RH, Akbari H, van Lenthe H, Kulik W, Wanders RJ, Frentzen M, et al. Identification and characterization of human cardiolipin synthase. FEBS Lett. 2006;580(13):3059-64.

31. Yoshimura Y, Araki A, Maruta H, Takahashi Y, Yamashita H. Molecular cloning of rat acss 3 and characterization of mammalian propionyl-CoA synthetase in the liver mitochondrial matrix. J Biochem. 2017;161(3):279-89.

32. Muniandy $\mathrm{M}$, Heinonen $\mathrm{S}$, Yki-Jarvinen $\mathrm{H}$, Hakkarainen $\mathrm{A}$, Lundbom J, Lundbom N, et al. Gene expression profile of subcutaneous adipose tissue in BMI-discordant monozygotic twin pairs unravels molecular and clinical changes associated with sub-types of obesity. Int J Obes. 2017:41(8):117684.

33. Bassols J, Botas P, Moreno-Navarrete JM, Delgado E, Ortega F, Ricart W, et al. Environmental and genetic factors influence the relationship between circulating IL-10 and obesity phenotypes. Obesity (Silver Spring, Md). 2010; 18(3):611-8

34. Wang W, Jiang W, Hou L, Duan H, Wu Y, Xu C, et al. Weighted gene coexpression network analysis of expression data of monozygotic twins identifies specific modules and hub genes related to BMI. BMC Genomics. 2017;18(1):872

35. Gao Z, Hwang D, Bataille F, Lefevre M, York D, Quon MJ, et al. Serine phosphorylation of insulin receptor substrate 1 by inhibitor kappa B kinase complex. J Biol Chem. 2002;277(50):48115-21.

36. Hirosumi J, Tuncman G, Chang L, Gorgun CZ, Uysal KT, Maeda K, et al. A central role for JNK in obesity and insulin resistance. Nature. 2002;420(6913): 333-6.

\section{Publisher's Note}

Springer Nature remains neutral with regard to jurisdictional claims in published maps and institutional affiliations.

\section{Ready to submit your research? Choose BMC and benefit from:}

- fast, convenient online submission

- thorough peer review by experienced researchers in your field

- rapid publication on acceptance

- support for research data, including large and complex data types

- gold Open Access which fosters wider collaboration and increased citations

- maximum visibility for your research: over $100 \mathrm{M}$ website views per year

At BMC, research is always in progress.

Learn more biomedcentral.com/submissions 